\title{
Soft, Rotating Pneumatic Actuator
}

\section{Citation}

Ainla, Alar, Mohit S. Verma, Dian Yang, and George M. Whitesides. 2017. “Soft, Rotating Pneumatic Actuator." Soft Robotics (May 30). doi:10.1089/soro.2017.0017.

\section{Published Version}

10.1089/soro.2017.0017

\section{Permanent link}

http://nrs.harvard.edu/urn-3:HUL.InstRepos:34708428

\section{Terms of Use}

This article was downloaded from Harvard University's DASH repository, and is made available under the terms and conditions applicable to Open Access Policy Articles, as set forth at http:// nrs.harvard.edu/urn-3:HUL.InstRepos:dash.current.terms-of-use\#OAP

\section{Share Your Story}

The Harvard community has made this article openly available.

Please share how this access benefits you. Submit a story.

Accessibility 


\title{
Soft, Rotating Pneumatic Actuator
}

\author{
Alar Ainla $^{1+}$, Mohit S. Verma ${ }^{1+}$, Dian Yang $^{2+}$ and George M. Whitesides ${ }^{1,3,4 *}$ \\ ${ }^{1}$ Department of Chemistry and Chemical Biology, Harvard University, 12 Oxford Street, \\ Cambridge, MA 02138, USA.
}

${ }^{2}$ School of Engineering and Applied Sciences, Harvard University, 29 Oxford Street, Cambridge, MA 02138, USA.

${ }^{3}$ Wyss Institute for Biologically Inspired Engineering, Harvard University, 60 Oxford Street, Cambridge, MA 02138, USA.

${ }^{4}$ Kavli Institute for Bionano Science and Technology, Harvard University, 29 Oxford Street, Cambridge, MA 02138, USA.

${ }^{+}$Authors contributed equally

*Author to whom correspondence should be addressed: gwhitesides@gmwgroup.harvard.edu 


\begin{abstract}
This paper describes a soft pneumatic actuator that generates cyclical motion. The actuator consists of several (three, four, or five) chambers (arranged around the circumference of a circle surrounding a central rod) that can be actuated independently using negative pressure (or partial vacuum). Sequential actuation of the four-chamber device using reduced pressure moves the central rod cyclically in an approximately square path. We characterize the trajectory of the actuator and the force exerted by it, as we vary the material used for fabrication, the number of chambers, and the size of the actuator. We demonstrate two applications of this actuator: to deliver fluid while stirring (by replacing the central rod with a needle), and for locomotion that mimics a reptilian gait (by combining four actuators together).
\end{abstract}

\title{
Introduction
}

Soft and hard actuators - as they are developing — seem to have different and complementary capabilities. ${ }^{1-19}$ Hard actuators, for example, are superior when high force, accuracy of positioning, repeatability, and speed of actuation are required. Soft actuators are more directly compatible with tasks requiring delicacy, adaptability, collaborativity (meaning the ability to operate safely with humans), resistance to damage by impact, and light weight. ${ }^{1-6,20,21}$ That complementarity in characteristics is not exclusive, and there are types of motion required by both soft and hard systems: one of these is what we will call "cyclical" motions.

Cyclical motion in a circular trajectory is very easily accomplished in hard systems using a wheel or ring, an axel, and bearings that allow motion of the axel relative to the bearing: the wheels in automobiles or the hands in an analog clock are examples. For soft systems, the design generating an analogous function is not immediately obvious. What would be a corresponding 
system — one capable of pointing in any direction relative to a central axis — for a device that does not have a wheel, axel, or bearings? There are a number of possible solutions for this problem. Here, we describe one, based on a circular array of pneumatically actuated subcomponents - which we call a cyclical vacuum-actuated machine (cVAM, Figure 1). Mazzeo et al. have recently described a system that is related in concept, although different in execution and application. $^{22}$

Prior work ${ }^{4,22-29}$ on soft actuators has suggested a number of approaches related to what we describe here. Wait et al. developed a spherical rolling robot using inflatable rubber bladders. ${ }^{23}$ Rus et al. demonstrated the coordinated use of pairs of soft bending actuators to achieve rolling motion. ${ }^{24}$ Mazzolai et al. demonstrated the use of shape memory alloys to mimic tentacles of octopus. ${ }^{25}$ We demonstrated pneumatic tentacles capable of pointing in any direction in the plane of their base (and by extension of the principle on which they operate) in directions spanning more than 90 degrees from the perpendicular to that plane. ${ }^{4}$ We also demonstrated (in the course of research focused more in fabrication than actuation) structures ("click-e-bricks") capable of limited directional pointing. ${ }^{28}$ Rus et al. made a soft pneumatic belt that can roll by sequential actuation of the actuators arranged in a ring. ${ }^{26}$ Lazarus and Reis used an auxetic design to achieve bending and twisting in a cylindrical actuator. ${ }^{29}$ Mazzeo et al. demonstrated that sequential inflation of actuators positioned around the circumference of a circle can produce rotational motion; they thus created soft wheels. ${ }^{22}$

- The structure in Figure 1 follows principles related to the pneumatic wheel of Mazzeo et al., but has three key differences. (i) A cVAM acts as a single part (because the central rod and the surrounding chambers are joined). Mazzeo's rotary actuator has two parts-a stator and a rotor. (ii) A cVAM uses negative pressure (vacuum) as the pneumatic source of power, as 
opposed to positive pressure; it thus, has no risk of bursting from overpressure, but also a different relation between power output and size/structure. (iii) A cVAM is analogous to a joystick, while Mazzeo's rotary actuator is similar to a stepper motor. The joystick design allows more degrees of freedom in actuation than a stepper motor, but also places greater demands on the part of the structure where "revolution" (perhaps better described in cVAMs as "flexing" or "wobbling") occurs. Other technical differences are summarized in Table

Table 1.

\section{Results and Discussion}

\section{Schematics and operation of cVAM}

A cVAM consists of four soft pneumatic chambers organized in a ring; these surround and steer a central rod whose top moves approximately in a 2-D plane (see Figure 1 and Movie S1). The cVAM is fabricated by molding elastomers (using 3D-printed parts) and positioning a rigid rod (in Figure 1, a wooden dowel) in the center of the device by curing the elastomer around its base (Figure S1). When a differential pressure of $-90 \mathrm{kPa}$ (partial vacuum inside the chambers) is applied to two adjacent chambers, the rod deflects from the vertical by approximately $16^{\circ}$ (Figure $1 \mathrm{~B}$ ). Figure $1 \mathrm{C}$ illustrates various positions that can be attained by the rod by sequential actuation of adjacent chambers.

\section{Characterizing the torque obtained from cVAMs}

We fabricated cVAMs using elastomers (Table S1) with different values of stiffness (as characterized by their elastic modulus) and characterized their actuation with and without load using the setup illustrated in Figure 2A, where we evacuate two of the four chambers to move the central rod against gravity. Figure 2B shows a time-averaged image obtained for a typical 
actuator (as observed from a side view) with a load at the tip of the rod. The pressure difference between the inside and outside of the chambers was varied using a custom-built electronic pressure regulator (see supplementary information for details on assembly and operation) over the range of 0 to $-90 \mathrm{kPa}$ in increments of $\sim 4 \mathrm{kPa}$ to obtain Figure $2 \mathrm{C}$. As expected, actuators fabricated in stiffer elastomers show a smaller deflection of the rod at the same negative pressure. The actuator fabricated in 1:1 v/v mixture of Dragonskin:PDMS does not actuate completely under atmospheric conditions because the pressure difference is limited, but it could be useful for hyperbaric applications (such as deep-sea exploration).

When a load is added to the tip of the rod, Figure 2D shows the maximum angle of actuation (as illustrated in Figure 2A) for actuators fabricated in different elastomers (when evacuated at $-90 \mathrm{kPa}$ ). The slopes of the lines in this plot (Table S2) confirm that stiffer elastomers are less affected by the addition of load than softer elastomers. The deflection of the rod without applied negative pressure and the work done by the actuators are presented in Figure S3.

Scaling the size (by $2 \mathrm{x}$ or $3 \mathrm{x}$ ) of the actuator shows the expected trends in its performance as indicated in Figure S4 and explained in the Analysis section of supplementary information.

\section{Dynamic characterization of cVAM}

We also characterized the dependence of the angle of deflection (i.e. the angle between the initial position of the rod and its position upon actuation) of the rod on the frequency of revolution (number of revolutions per minute, or rpm, where one revolution is completed when the tip of the rod follows a trajectory that returns it to its initial position). By tracking the trajectory of the tip of the rod (see Movie S3 for a typical video used for characterization), Figure 3 illustrates that the rod follows a trajectory whose shape is governed by the number of chambers (i.e. it will form 
a triangle, a square, or a pentagon, for three, four, or five chambers respectively). One revolution involves six, eight, or ten steps for three, four, or five chambers as illustrated in Figure 3A. The frequency of revolution is controlled by changing how long the rod is held at each of these steps (we will refer to this time as hold time). When two adjacent chambers of the cVAM are actuated, the rod is at the corner of its respective shape; actuating a single chamber brings the rod approximately halfway around the edge of its respective shape. The shape of the trajectory is relatively constant as the hold time changes from $200 \mathrm{~ms}$ to $50 \mathrm{~ms}$ (Figure 3A) and the maximum angle of deflection (Figure 3B) decreases more severely $\left(\sim 0.005^{\circ} / \mathrm{rpm}\right)$ for three- and fourchamber actuators than for the five-chamber actuator $(\sim 0.001 \% \mathrm{rpm})$. As we increase the frequency of revolution further (hold time of $5 \mathrm{~ms}$ ), the trajectory starts to become more circular (Figure 3A). While the actuator functions with different numbers of chambers, the four-chamber design provides the most consistent trajectory over a wide range of frequencies of operation (Figure 3). Similar trends are observed when the actuator is scaled in size (Figure S5), but the larger actuator ( $3 \mathrm{x}$ scaled) shows a more dramatic drop in the angle of deflection $\left(\sim 0.010^{\circ} / \mathrm{rpm}\right)$ as a function of frequency of revolution.

When two chambers are actuated simultaneously, the angle of deflection is approximately twice that achieved by a single chamber (Figure S6) and the hysteresis is negligible as the negative pressure is ramped up or down (Figure S6).

\section{Characterizing cyclical motion in a fluid}

We used the actuator fabricated in Ecoflex 00-30 at 1x scale (Figure S1), with four chambers to characterize the cyclical motion in a fluid. Figure 4A shows a schematic of the setup used to characterize the motion of cVAMs in fluids. Three fluids with different viscosities were used to characterize it: air ( $\eta \approx 0.02 \mathrm{mPa} . \mathrm{s})$, water $(\eta \approx 0.89 \mathrm{mPa} . \mathrm{s}), 2 \% \mathrm{w} / \mathrm{v}$ carboxymethylcellulose 
(CMC, $\eta \approx 2300 \mathrm{mPa} . \mathrm{s})$ in water. Figure 4B shows sample images of the side view and bottom view of the stirrer. We operated the cVAMs at frequencies from $7.5 \mathrm{rpm}$ to $750 \mathrm{rpm}$ by changing the hold time (ranging from $1000 \mathrm{~ms}$ to $10 \mathrm{~ms}$ ) between each of the eight steps (Figure 3A) that make up one complete revolution.

As expected, the cVAMs produce a periodic response (Figure $4 \mathrm{C}$ ), the frequency of revolution increases as the frequency of operation of its pneumatic valves increases, while the amplitude of oscillation is approximately constant $( \pm 10 \%)$. At a frequency of revolution of 375 rpm (hold time of $20 \mathrm{~ms}$ ), the amplitude of oscillation increases (by $\sim 60 \%$ ), probably because the actuator is operating closer to resonance frequency. When the frequency of revolution is $750 \mathrm{rpm}$ (hold time of $10 \mathrm{~ms}$ ), the amplitude drops considerably (from $\sim 4 \mathrm{~mm}$ to $\sim 1 \mathrm{~mm}-$ a decrease of $\sim 80 \%$ ). In comparison to the setup used in Figure 3, here we have added a load (a 2.4-g spherical rubber ball) to a needle (instead of a wooden dovel, such that fluid could be delivered or extracted as shown in Figure 5) and thus, the response time (time between the signal from the solenoid valve and the movement of the ball) of the cVAM increases. Thus, a hold time of $10 \mathrm{~ms}$ is insufficient to achieve complete movement of the central rod. We also observe that at the end of each of the eight steps of the revolution, the stirrer oscillates (with a decay) due to elasticity of the cVAMs (Figure 4 D). We can use these decaying oscillations to estimate the angular spring constant of the actuator as $0.0045 \mathrm{~N} \cdot \mathrm{m}_{\mathrm{rad}} \mathrm{ra}^{-1}$ and the angular damping constant due to the elastomer in air as $3.9 \times 10^{-5}$ N.m.s.rad ${ }^{-1}$ (see details in supporting information). The damping of the amplitude of this oscillation can be affected by the viscosity of the fluid, if the viscosity is high enough. For example, when the viscosity of the fluid is significantly higher (2300 mPa.s) than water $(0.89 \mathrm{mPa} . \mathrm{s})$, then the oscillator operates in an overdamped regime (Figure $4 \mathrm{E})$. 
Using the viscous drag force exerted by the fluids, we calculated the power output of the actuator as a function of frequency of revolution (Figure $4 \mathrm{~F}$, details of calculations are in the supporting information). For the different frequencies of revolution, the work done per revolution by the actuator on the fluid remains approximately constant (because the force and displacement are the same for each step for the different frequencies of revolution). As the frequency of revolution increases, the time period over which this work is done decreases, and hence the power dissipated while stirring increases. As expected, frequency of revolution and power are linearly related (Figure $4 \mathrm{~F}$ ). The only exception is when the hold time (10 ms, with frequency of revolution of $750 \mathrm{rpm}$ ) for each step is shorter than the time interval required for the actuator to respond. In this regime, incomplete actuation leads to a decrease in the dissipated power.

Figures S2 and S3 characterize the performance of the actuator when a load is applied at one end of the rod. These characteristics can be used to estimate the spring constant at 0.0095 N.m.rad ${ }^{-1}$, which is about double compared to the previous estimate $\left(0.0045 \mathrm{~N} \cdot \mathrm{m} \cdot \mathrm{rad}^{-1}\right)$. Additionally, the thermodynamic efficiency is approximately $2 \%$ and the power dissipated is about $2.3 \mathrm{~mW}$ (see supplementary information for details on calculations; for comparison, we have reported a soft linear actuator showing efficiency of approximately $27 \%,{ }^{20}$ and biological muscles have efficiency of about $20-40 \%{ }^{30,31}$ ). These characteristics could be improved by optimizing the design of the actuator, for example, by making the external walls thicker to minimize losses of energy by compression of the actuator.

\section{Using cVAMs for delivering fluid while stirring}

We replaced the central wooden dowel with a stainless steel needle $(16 \mathrm{G}, 10 \mathrm{~cm})$ and connected the needle to a tube (Tygon ${ }^{\circledR}$ S3) for delivering fluids. We attached a spherical ball at the base of 
the needle (see Movie S2 for a demonstration of stirring) for two reasons: i) to increase the drag force in viscous fluid and hence, the stirring ability, and ii) to aid in characterization because a spherical shape simplifies force calculations (as discussed in the previous section). Since the cVAMs produce a cyclical motion without rotating the needle, it is possible to deliver fluids without entangling the tubes. Using fluorescein ( $1 \mathrm{mM}$ in water) as a model dye, we demonstrated that the cVAMs can be used to deliver fluid (at the rate of $10 \mathrm{~mL} / \mathrm{min}$ ) into a water bath $(150 \mathrm{~mL})$ while stirring at $375 \mathrm{rpm}$ (hold time of $20 \mathrm{~ms})$. Qualitatively, Figure 5Error! Reference source not found. and Movie S4 show that stirring using cVAMs can achieve a homogenous solution (i.e. fluorescein is dispersed throughout the water bath) within 10 seconds while delivering fluorescein continuously. On the contrary, when the cVAM is not stirring, the delivery of fluorescein is heterogeneous even after 20 s (i.e. the convection created by stirring by cVAM is considerably more effective for mixing than diffusion alone, Figure 5Error! Reference source not found.).

\section{Using cVAMs for locomotion}

Actuating the ring of pneumatic chambers in clockwise or counter-clockwise sequence causes the tip of the central rod to move in a cyclical fashion while following an approximately square trajectory (Figure 3). We took advantage of this motion to build a quadrupedal soft robot that uses this cyclical movement to walk (Figure 6). In designing a gait, we mimicked the walking gait of a reptile. While walking, a reptile moves its diagonal limbs simultaneously (Figure S11 ${ }^{32}$ ) and so does our soft robot (see Movies S5, S6, S7).

\section{Conclusions}

- cVAMs offer a new method to generate a cyclical motion using a soft actuator. It is useful to compare the cVAM with the related device described by Mazzeo et al. ${ }^{22}$ Four advantages can be 
listed. (i) The cVAMs do not require a lubricant. (ii) They cannot burst at actuation pressures above their operating specifications because they operate under negative pressure (partial vacuum) rather than positive pressure. (iii) The frequency of revolution is controlled by the hold time between steps while the negative pressure (in the chambers) controls the shape of the trajectory; Mazzeo's rotational actuator requires a critical pressure below which there is no rotation because the inflation of the chambers depends on the pressure applied (and if the pressure is too low, the sequential inflation of chambers does not lead to rotation). This relationship allows us to achieve rapid revolutions ( $\sim 750 \mathrm{rpm}$ without altering the trajectory) compared to Mazzeo et al. ( $\sim 20$ rpm at a comparable pressure difference of $90 \mathrm{kPa}$ ). (iv) cVAMs contract rather than expand when being used, and thus can be used in space-constrained environments. The elastomers are less strained when operated using reduced pressure relative to operation under positive pressure and thus, can have increased lifetime (we tested cVAMs with $10^{6}$ cycles and the actuation remained unchanged, as seen in Figure S12). cVAMs exhibit three limitations. i) Under ambient conditions, the pressure difference is limited to approximately $100 \mathrm{kPa}$ because cVAMs actuate using vacuum. This property governs the force that can be applied by the cVAMs. The limitation might become an advantage in hyperbaric conditions such as in deep-sea applications. ii) Operation of the cVAM involves many cycles of low amplitude stretching and compressing of the elastomer "hub," and failure in this region is a possibility (although unlikely for at least a million cycles, as seen in Figure S12). iii) cVAMs are not directly suitable for causing an axle and wheel to rotate because the central rod does not actually rotate, instead it follows an approximately square trajectory. Table 
Table 1 summarizes additional technical differences.

The cVAMs can be used to deliver fluids, or to sample, while stirring without entangling the tubes used for delivery (or sampling) as demonstrated by the stirring of fluorescein in Figure 5Error! Reference source not found.. Since the actuators are pneumatic, cVAMs can be used in operations that are sensitive to electromagnetic fields, or use flammable liquids, where unshielded electric motors could cause interference or explosion.

These cVAMs can be coupled to each other to mimic some parts of the motion used by animals (reptiles) to move their limbs in walking. In reptilian walking, several joints function together in each step. One characteristic of this gait is that diagonal limbs move simultaneously (or almost simultaneously); ${ }^{32}$ we implemented this diagonal co-ordination in our crude biomimetic model.

\section{Acknowledgments}

DY's work on biomimetic design was funded by a subcontract from Northwestern University under DOE award number DE-SC0000989. Work on mechanics and characterizations of the actuator were funded by the DOE, Division of Materials Sciences and Engineering, grant number ER45852. AA was funded by the Swedish Research Council (VR) postdoctoral fellowship. MSV was funded by the Banting Postdoctoral Fellowship from the Government of Canada. Jim MacArthur and The Electronic Instrument Design Lab (Department of Physics, Harvard University) provided an excellent environment for the development of the electronic parts of the system.

\section{Author contributions}


A.A., M.S.V., D.Y., and G.M.W. designed the experiments. A.A. and D.Y. executed the experiments. D.Y. designed and fabricated the actuators. A.A. and D.Y. designed and fabricated the quadrupedal robots. M.S.V., D.Y., and A.A. analyzed the results. M.S.V., A.A., D.Y., and G.M.W. wrote the manuscript.

The authors declare no conflict of interest. 


\section{References}

1. Suzumori K, Iikura S, Tanaka H. Development of Flexible Microactuator and Its Applications to Robotic Mechanisms. Proc 1991 IEEE Int Conf Robotics and Automation. 1991; 2: 1622-1627.

2. Suzumori K, Iikura S, Tanaka H. Flexible Microactuator for Miniature Robots. 1991 Proc IEEE Micro Electro Mechanical Systems. 1991: 204-209.

3. Ilievski F, Mazzeo AD, Shepherd RF, Chen X, Whitesides GM. Soft Robotics for Chemists. Angew Chem Int Ed. 2011; 50(8): 1890-1895.

4. Martinez RV, Branch JL, Fish CR, Jin L, Shepherd RF, Nunes RM, Suo Z, Whitesides GM. Robotic Tentacles with Three-Dimensional Mobility Based on Flexible Elastomers. Adv Mater. 2013; 25(2): 205-212.

5. Mosadegh B, Polygerinos P, Keplinger C, Wennstedt S, Shepherd RF, Gupta U, Shim J, Bertoldi K, Walsh CJ, Whitesides GM. Pneumatic Networks for Soft Robotics That Actuate Rapidly. Adv Funct Mater. 2014; 24(15): 2163-2170.

6. Rus D, Tolley MT. Design, Fabrication and Control of Soft Robots. Nature. 2015; 521(7553): 467-475.

7. Trivedi D, Rahn CD, Kier WM, Walker ID. Soft Robotics: Biological Inspiration, State of the Art, and Future Research. Appl Bionics Biomech. 2008; 5(3).

8. Pfeifer R, Lungarella M, Iida F. The Challenges Ahead for Bio-Inspired 'Soft' Robotics. Commun ACM. 2012; 55(11): 76-87.

9. Kim S, Laschi C, Trimmer B. Soft Robotics: A Bioinspired Evolution in Robotics. Trends Biotechnol. 2013; 31(5): 23-30.

10. Trimmer B. Soft Robots. Current Biology. 2013; 23(15): R639-R641.

11. Bauer S, Bauer-Gogonea S, Graz I, Kaltenbrunner M, Keplinger C, Schwodiauer R. 25th Anniversary Article: A Soft Future: From Robots and Sensor Skin to Energy Harvesters. Adv Mater. 2014; 26(1): 149-162. 
12. Majidi C. Soft Robotics: A Perspective-Current Trends and Prospects for the Future. Soft Robot. 2014; 1(1): 5-11.

13. Laschi C, Cianchetti M. Soft Robotics: New Perspectives for Robot Bodyware and Control. Front Bioeng Biotechnol. 2014; 2(3).

14. Lu NS, Kim DH. Flexible and Stretchable Electronics Paving the Way for Soft Robotics. Soft Robot. 2014; 1(1): 53-62.

15. Jaeger HM. Celebrating Soft Matter's 10th Anniversary: Toward Jamming by Design. Soft Matter. 2015; 11(1): 12-27.

16. Marchese AD, Katzschmann RK, Rus D. A Recipe for Soft Fluidic Elastomer Robots. Soft Robot. 2015; 2(1): 7-25.

17. Whitesides GM. Bioinspiration: Something for Everyone. Interface Focus. 2015; 5(4).

18. Hughes J, Culha U, Giardina F, Guenther F, Rosendo A, Iida F. Soft Manipulators and Grippers: A Review. Front Robot AI. 2016; 3.

19. Zolfagharian A, Kouzani AZ, Khoo SY, Moghadam AAA, Gibson I, Kaynak A. Evolution of 3D Printed Soft Actuators. Sensor Actuat A-Phys. 2016; 250: 258-272.

20. Yang D, Verma MS, So J-H, Mosadegh B, Keplinger C, Lee B, Khashai F, Lossner E, Suo Z, Whitesides GM. Buckling Pneumatic Linear Actuators Inspired by Muscle. Adv Mater Technol. 2016; 1(3): 1600055.

21. Yang D, Verma MS, Lossner E, Stothers D, Whitesides GM. Negative-Pressure Soft Linear Actuator with a Mechanical Advantage. Adv Mater Technol. 2016; 2(1): 1600164.

22. Gong X, Yang K, Xie J, Wang Y, Kulkarni P, Hobbs AS, Mazzeo AD. Rotary Actuators Based on Pneumatically Driven Elastomeric Structures. Adv Mater. 2016; 28(34): 75337538.

23. Wait KW, Jackson PJ, Smoot LS. Self Locomotion of a Spherical Rolling Robot Using a Novel Deformable Pneumatic Method. IEEE Int Conf Robot. 2010: 3757-3762. 
24. Marchese AD, Onal CD, Rus D. Soft Robot Actuators Using Energy-Efficient Valves Controlled by Electropermanent Magnets. 2011 IEEE/RSJ Int Conf Intelligent Robots and System (IROS). 2011: 756-761.

25. Mazzolai B, Margheri L, Cianchetti M, Dario P, Laschi C. Soft-Robotic Arm Inspired by the Octopus: Ii. From Artificial Requirements to Innovative Technological Solutions. Bioinspir Biomim. 2012; 7(2).

26. Soft Autonomous Materials_-Using Active Elasticity and Embedded Distributed Computation (Experimental Robotics: The 12th International Symposium on Experimental Robotics) Correll N, Önal ÇD, Liang H, Schoenfeld E, Rus D Berlin, Heidelberg; Springer Berlin Heidelberg: 2014.

27. Morin SA, Kwok SW, Lessing J, Ting J, Shepherd RF, Stokes AA, Whitesides GM. Elastomeric Tiles for the Fabrication of Inflatable Structures. Adv Funct Mater. 2014; 24(35): 5541-5549.

28. Morin SA, Shevchenko Y, Lessing J, Kwok SW, Shepherd RF, Stokes AA, Whitesides GM. Using "Click-E-Bricks" to Make 3d Elastomeric Structures. Adv Mater. 2014; 26(34): 5991-5999.

29. Lazarus A, Reis PM. Soft Actuation of Structured Cylinders through Auxetic Behavior. Adv Eng Mater. 2015; 17(6): 815-820.

30. Madden JDW, Vandesteeg NA, Anquetil PA, Madden PGA, Takshi A, Pytel RZ, Lafontaine SR, Wieringa PA, Hunter IW. Artificial Muscle Technology: Physical Principles and Naval Prospects. IEEE J Oceanic Eng. 2004; 29(3): 706-728.

31. Huber JE, Fleck NA, Ashby MF. The Selection of Mechanical Actuators Based on Performance Indices. Proc R Soc Lond A. 1997; 453(1965): 2185-2205.

32. Reilly SM, Delancey MJ. Sprawling Locomotion in the Lizard Sceloporus Clarkii: Quantitative Kinematics of a Walking Trot. J Exp Biol. 1997; 200(4): 753-765. 


\section{Table}

Table 1: Comparison of the rotary actuator developed by Mazzeo et al. and our cyclical vacuumactuated machine (cVAM)

\begin{tabular}{|c|c|c|}
\hline Property & $\begin{array}{l}\text { Mazzeo's } \\
\text { Rotary actuator } 22\end{array}$ & $\begin{array}{l}\text { Current work } \\
\text { cVAMs }\end{array}$ \\
\hline Actuation type & Rotation & $\begin{array}{l}\text { Angular deflection and } \\
\text { revolution }\end{array}$ \\
\hline Pneumatic source & $\begin{array}{l}\text { Pressure }(\sim 50 \mathrm{kPa} \text { to } 100 \\
\mathrm{kPa})\end{array}$ & Vacuum $(-5 \mathrm{kPa}$ to $-90 \mathrm{kPa})$ \\
\hline Degrees of freedom & 1 (angle) & 2 (angle, radius) \\
\hline Drive mode & Digital & Digital or analog \\
\hline Construction & 2 parts (stator and rotor) & 1 part (monolithic) \\
\hline Analogy & Stepper motor & Joystick \\
\hline Number of compartments & 16 & 3,4 , or 5 \\
\hline $\begin{array}{l}\text { Number of independent } \\
\text { pneumatic inputs }\end{array}$ & 4 & 3,4, or 5 \\
\hline $\begin{array}{l}\text { Number of steps in one full } \\
\text { rotation or revolution }\end{array}$ & 16 & $3,4,5$, or $6,8,10$ \\
\hline Frequency range & $\sim 14-18 \mathrm{rpm}$ & $\sim 10-1000 \mathrm{rpm}$ \\
\hline Power output & $8.4 \mathrm{~mW}$ or $26 \mathrm{~mW}$ & $2.3 \mathrm{~mW}$ \\
\hline Thermodynamic efficiency & not reported & $\sim 2 \%$ \\
\hline Lifetime & not reported & $>10^{6}$ cycles \\
\hline
\end{tabular}




\section{Figures}
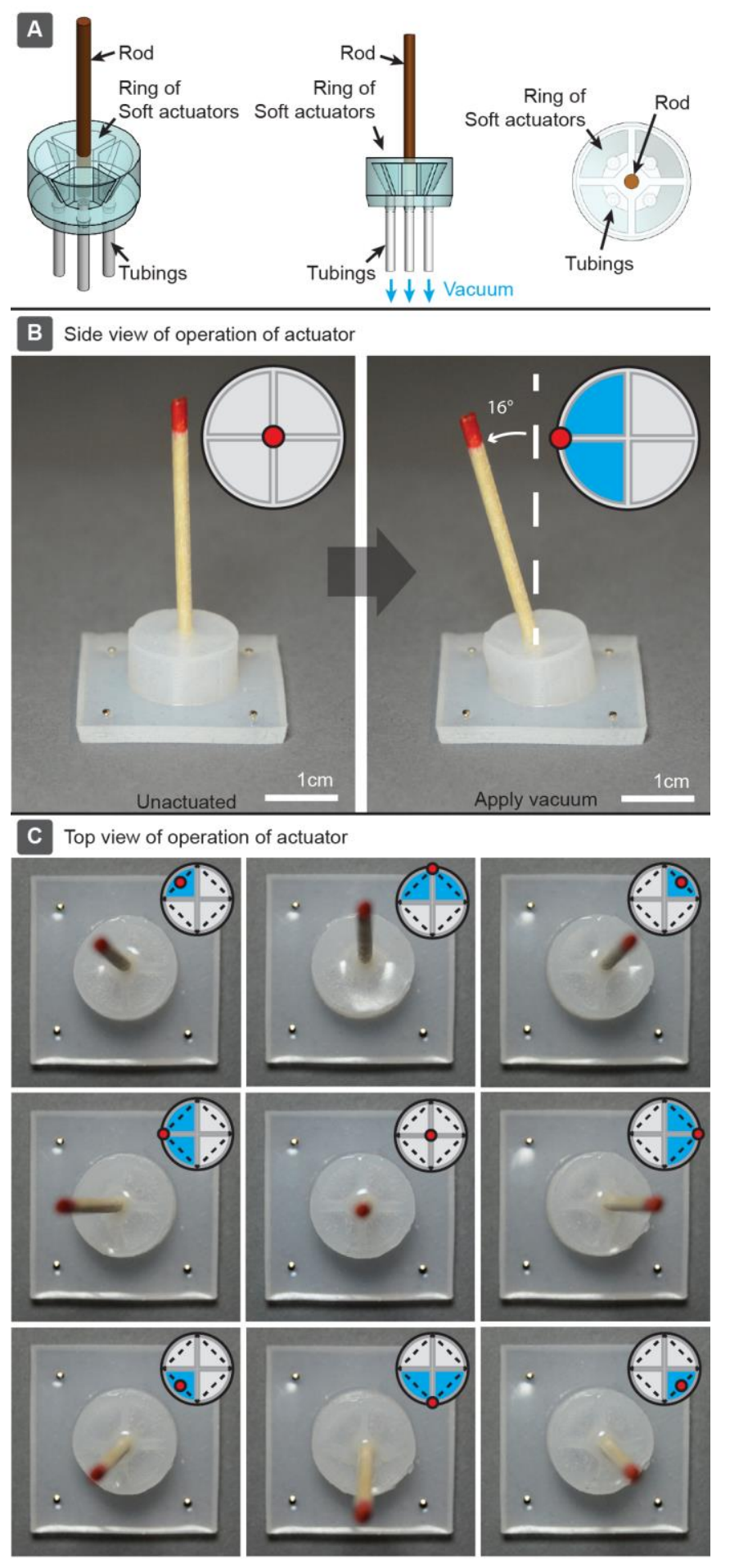

Figure 1: a) Schematics showing the design of a cyclical vacuum-actuated machine (cVAM). A typical cVAM unit contains four soft pneumatic air chambers that can pull the central hard rod in 
different directions upon application of vacuum. b) Images of a cVAM unit where the left two neighboring chambers are actuated at the same time by applying a negative pressure of $-90 \mathrm{kPa}$ (marked by blue shading in the schematic), tilting the rod (shown as a red circle in the schematic) in the middle. The tubing is fed through a hole on the grey platform, which is covered by the actuator and hidden from view. Scale bars are $1 \mathrm{~cm}$. c) Eight distinct directions are achieved by applying vacuum to one or two neighboring air chambers. A neutral position is shown in the middle when no vacuum is applied. 

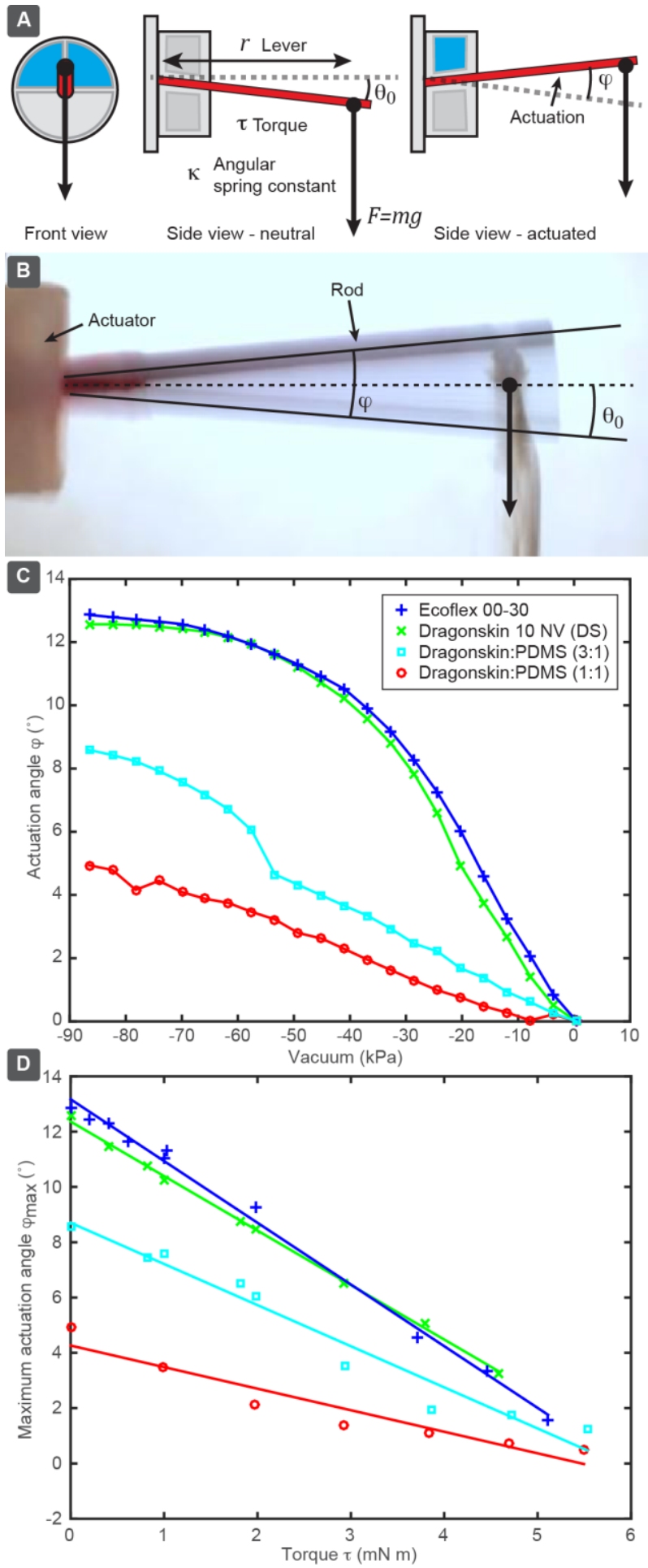

Figure 2: The effect of materials used for fabricating the actuators (all actuators are of the same size and have four chambers). (A) Schematic of the setup used for characterization: two 
chambers (highlighted with blue shading) were actuated and the actuator was used to lift weight. The negative pressure (relative to atmosphere) was ramped continuously with an electronic pressure control system as described in the supplementary information. The actuation angle $\varphi$ was recorded using a camera from the side. (B) Time-averaged actuation as seen from the side. (C) Actuation angle as a function of applied negative pressure for actuators made out of different materials (no load was applied, $\tau=0$ ). Initial slopes (in the linear regime at relatively small actuation angles) are $\sim 0.28^{\circ} / \mathrm{kPa}, \sim 0.09^{\circ} / \mathrm{kPa}$ and $\sim 0.06^{\circ} / \mathrm{kPa}$ respectively. (D) Maximum actuation $\varphi_{\max }$ as a function of different loads (varying torque). The maximum actuation decreases with increased load but the effect is less severe for stiffer elastomers (they have smaller slope). Further characterization is provided in supplementary information. 


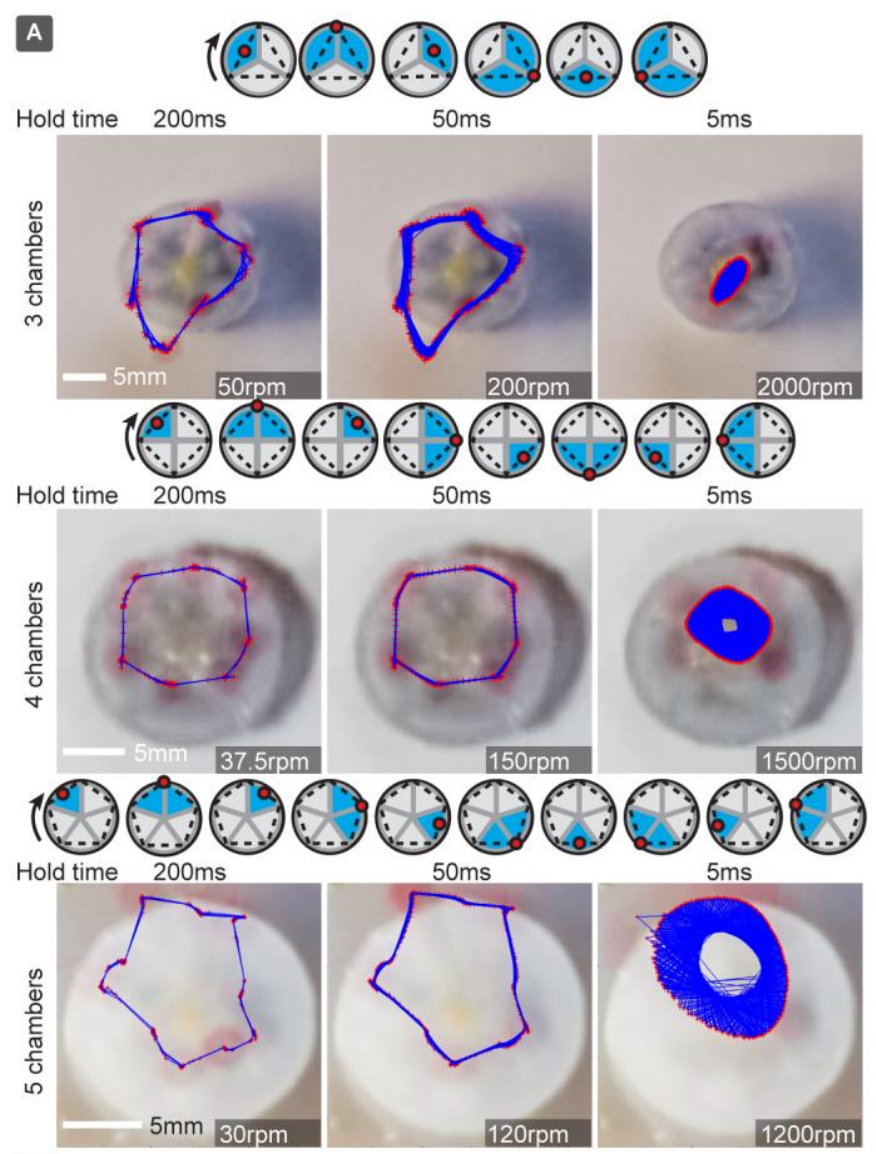

B

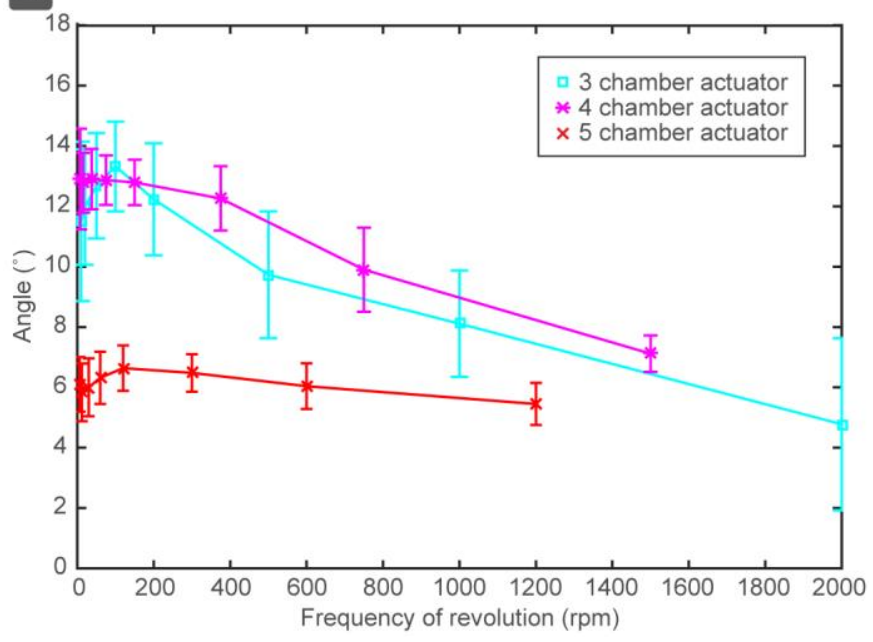

Figure 3: The effect of varying the number of chambers (three, four, or five) on the actuation of cVAMs. All actuators are made of same material (Ecoflex 00-30) and are of the same size. Volume of each chamber is $137 \mu \mathrm{L}, 99 \mu \mathrm{L}$, and $75 \mu \mathrm{L}$ for actuators with three, four, and five chambers respectively. (A) Schematic representation of the order of actuation of the chambers 
(six, eight, or ten steps per revolution), blue shows evacuated chambers, and red circle shows movement of tip position. Changes in the angle of deflection were obtained as a function of frequency of revolution (adjusted by changing the hold time at each step of the revolution). Images show three representative data points while the plot in part B) represents maximum deflected angle for the entire range of frequencies of revolution that were tested. (The trajectory of the rod is slightly off-center because the camera was not perfectly aligned with the center of the rod.) A pressure difference between the chambers and the atmosphere of $90 \mathrm{kPa}$ was used. The pictures presented here are time-averaged by overlaying frames from the corresponding video. The red marks show the location of the center of the rod as obtained from image tracking while blue lines indicate the trajectory. (B) Changes in the angle of deflection plotted as a function of the frequency of revolution. The values are reported as mean \pm S.D. obtained from the maximum deflection of the rod as the rod undergoes revolution at different frequencies (while being held at each frequency for $10 \mathrm{~s}$ and recorded in a video at $240 \mathrm{fps}$ ). 

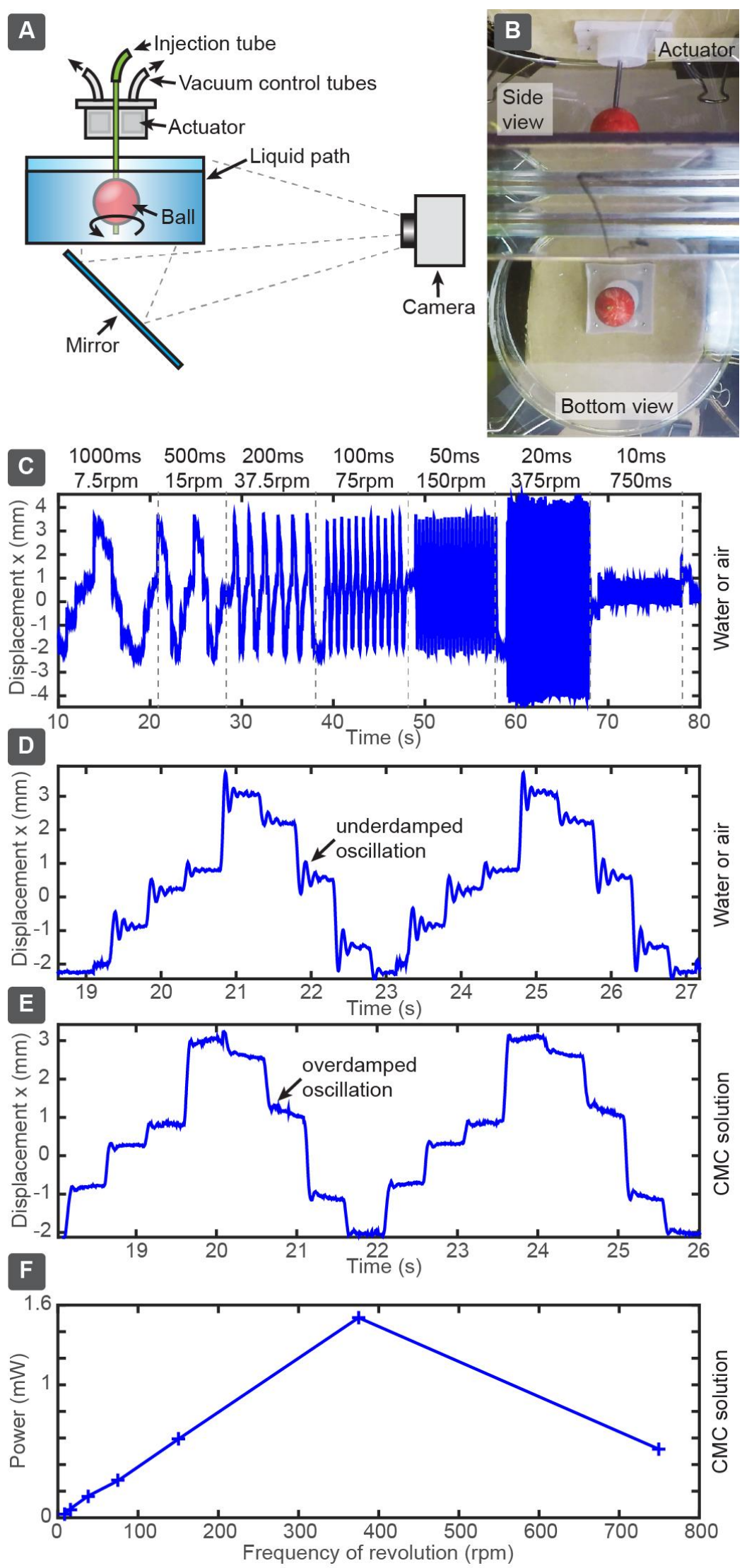

Page $\mathbf{2 3}$ of $\mathbf{2 6}$ 
Figure 4: Characterization of the cyclical actuation of cVAMs in fluids. (A) Schematic illustration of the experimental setup. (B) A sample image of the setup: the bottom view is obtained using a mirror placed at an angle. (C) Change in position of the ball as the frequency of revolution changes (by changing the hold time for each step of revolution; the hold time and frequency of revolution are shown above the plot). The oscillations are similar in air and water and also in the $\mathrm{x}$ and $\mathrm{y}$ directions because of symmetry. (D, E) Observations of underdamped oscillations (in water or air) and overdamped oscillations (in carboxymethylcellulose solution) for during each step of the revolution. (F) Average power output of the cVAMs (calculated based on the estimates of work done by the rubber ball in viscous fluid) at different frequencies of revolution. 


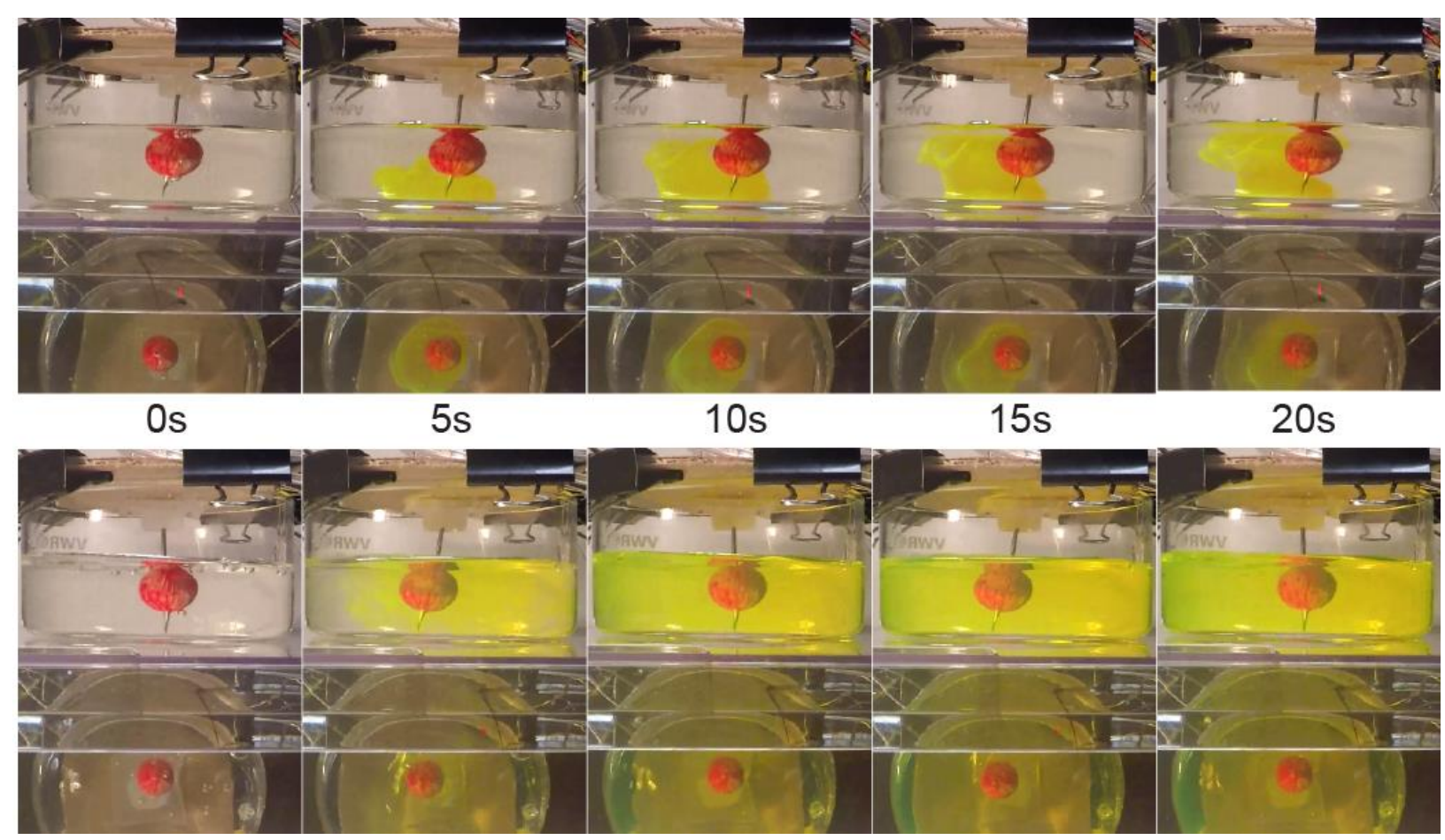

Figure 5: Demonstration of simultaneous delivery of fluid and stirring using cVAM. A solution of fluorescein (1 mM in water) is delivered (at the rate of $10 \mathrm{~mL} / \mathrm{min})$ into a bath $(150 \mathrm{~mL})$ of water while the cVAM is off (top sequence) or operating at $375 \mathrm{rpm}$ (bottom sequence). A video was captured (Movie S5) and then frames were extracted at different time points. Each frame shows the side view and bottom view (reflected using a mirror at an angle). 


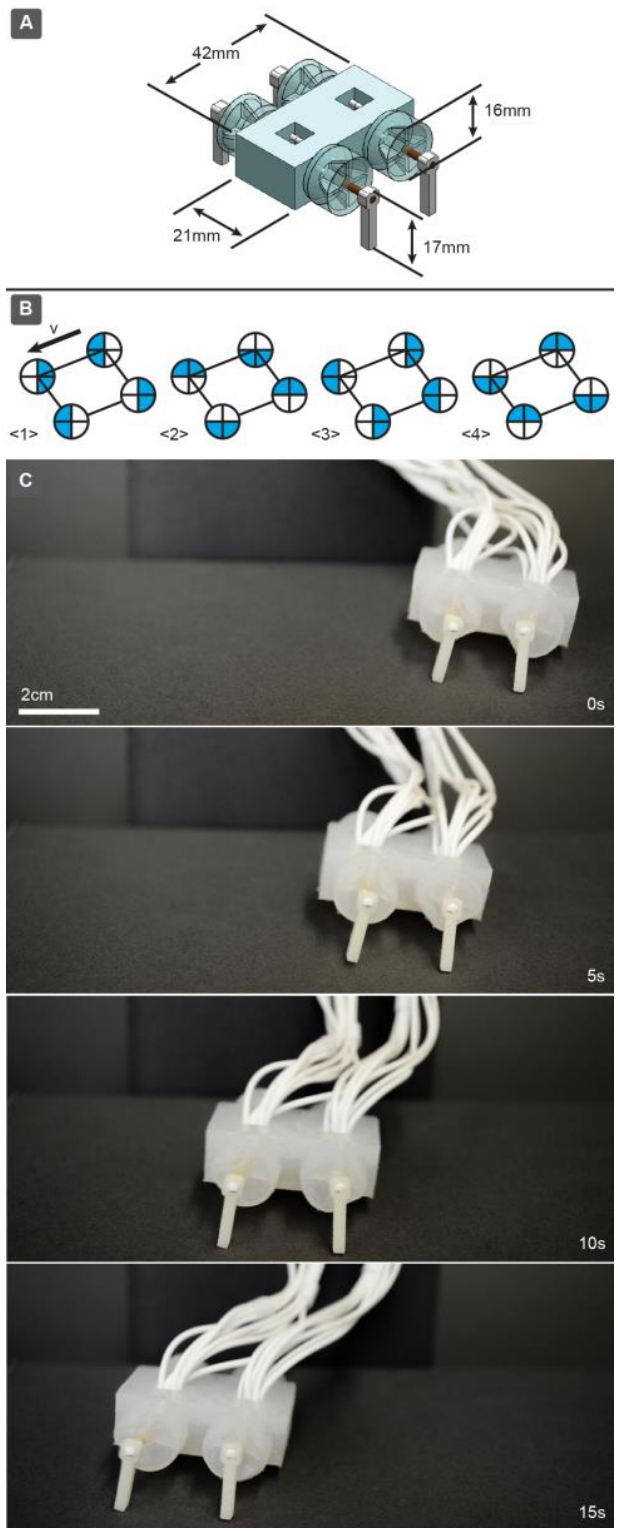

Figure 6: Demonstration of locomotion with cVAMs. a) A four-legged "walker" with four cVAMs to move its legs. b) Vacuum pulses are applied to four chambers on each leg in a cyclic sequence. A phase difference of $180^{\circ}$ exists between the front and back leg of each side, while two diagonal pairs of legs each have the same phase. c) The "walker" moves at $0.5 \mathrm{~cm} / \mathrm{s}$ (or 0.12 body length per second) when vacuum states are changed every $0.1 \mathrm{~s}$. 